\title{
Placental Pathologies in the Preterm Labors
}

\section{ART ICLE INF O}

\section{Article Type}

Original research

\section{Authors}

Sarafzadeh A* $M D$,

Jamalou M. ${ }^{1} P h D$,

Roustaei Z. ${ }^{2} P h D$

\section{How to cite this article} Sarafzadeh A, Jamalou M, Roustaei Z. Placental Pathologies in the Preterm Labors. Sarem Journal of Reproductive Medicine.2018;2(2) :59-64.

\section{A B S T R A C T}

Aims The placenta provides oxygen and nutrients to fetus and plays a central role in embryonic development. Abnormal growth of the placenta is associated with negative pregnancy outcomes. Preterm labor is one of the most important outcomes of placental abnormalities. The objective of this study was to investigate the pathology of placenta in preterm labor and prediction of neonate's outcomes.

Materials \& Methods This cross-sectional study was conducted on 118 placentas obtained from preterm labor cases in Sarem women's hospital (Tehran, Iran) during one year. At least 3 specimens were taken from each placenta and stained with $H \& E$ and examined by pathologists, microscopically. The data were analyzed by logistic regression analysis.

Findings No pathological changes were found in $89.5 \%$ of the placentas; however, $10.0 \%$ of the placentas showed pathological changes. Three parameters, including syncytial knots, chorangiosis and microcalcification were the most effective variables on pathological results. Accordingly, increase of one unit in syncytial knot raise the chance of preterm labor 0.01 fold. Besides, the change in the amount of chorangiosis can increase the chance of preterm labor 0.252 fold and changes in Microcalcification can raise the chance for preterm labor up to 0.14 fold on average.

Conclusion Syncytial knots, chorangiosis and microcalcification are the most effective pathological changes in the pathology of placenta in preterm labor. Keywords Placenta; Chorangiosis; Syncytial Knots; Pathological Changes; Microcalcification;
Preterm Labor

\section{I T A T I O N L I N KS}

[1] The role of placental examination and its pathology in obstetric risk management [2] Horseshoe Placenta and Preterm Labour: A Case Report [3] Association between battledore placenta and perinatal complications [4] Estimating the causes of 4 million neonatal deaths in the year 2000 [5] The role of prenatal care in recurrent preterm birth [6] The worldwide incidence of preterm birth: A systematic review of maternal mortality and morbidity [7] Preterm delivery in women with pregestational diabetes mellitus or chronic hypertension relative to women with uncomplicated pregnancies. The National institute of Child health and Human Development Maternal- Fetal Medicine Units Network [8] Investigating socio-economic disparities in preterm birth: Evidence for selective study participation and selection bias [9] Study of the Incidence, and Maternal and fetal risk factors for intra uterine fetal death [10] Abnormal placental findings associated with non-reassuring fetal monitoring and excellent neonatal outcomes [11] Placental pathology is associated with illness severity in preterm infants in the first twenty four hours after birth [12] Ballantyne syndrome: Is placental ischemia the etiology? [13] Placenta membranacea [14] Why all placentas should be examined by a pathologist in 1990 [15] Pathologic examination of the placenta: Recommended versus observed practice in a university hospital [16] Histopathological examination of the placenta: Key issues for pathologists and obstetricians. Pathology [17] Previous miscarriage and stillbirth as risk factors for other unfavourable outcomes in the next pregnancy [18] Efficacy of $17 \alpha$-hydroxyprogesterone caproate in preterm delivery prevention of preterm delivary [19] The role of preterm placental calcification in high-risk pregnancy as a predictor of poor uteroplacental blood flow and adverse pregnancy outcome [20] Exploring the relationship between preterm placental calcification and adverse maternal and fetal outcome [21] Effects of epithelial cells on amniotic membrane angiogenic properties using rat aortic ring assay [22] Chorangiosis of Chorionic Villi: What Does It Really Mean? [23] Syncytial knots and intervillous bridges in the human placenta: An ultrastructural study [24] Syncytial knots as a reflection of placental maturity: Reference values for 20 to 40 weeks gestational age

\section{Article History}

Received: January 15, 2017

Accepted: May 14, 2017

ePublished: June 15, 2018 


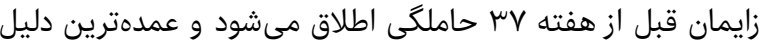

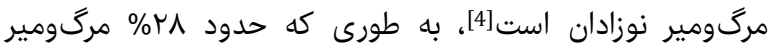

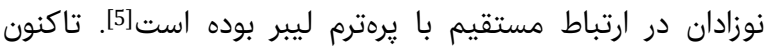

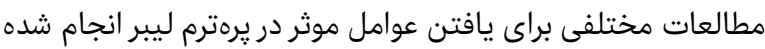

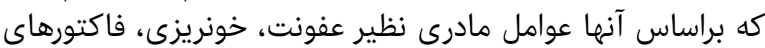

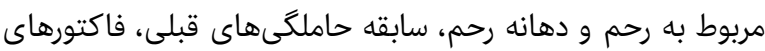

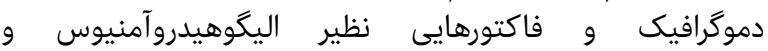

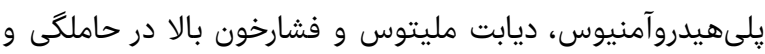

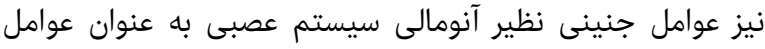

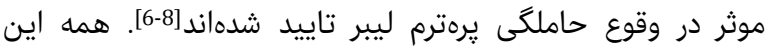
عوامل بر جفت هم اثر مستقيم دارند.

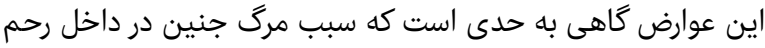

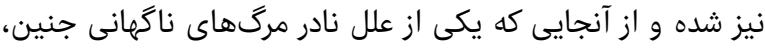

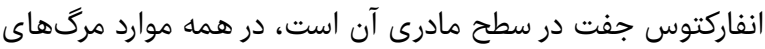

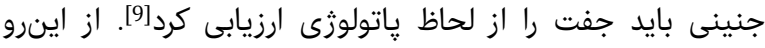

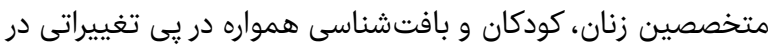

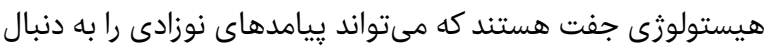

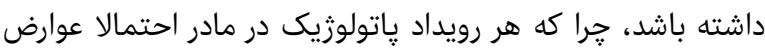

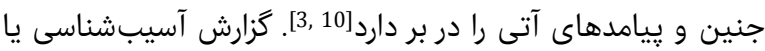

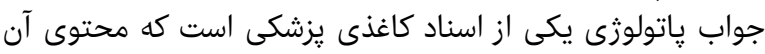

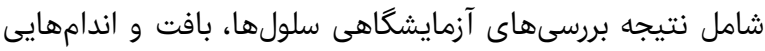

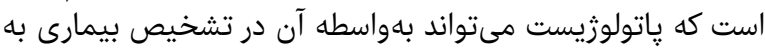

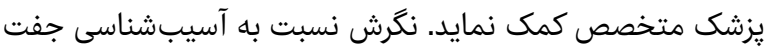

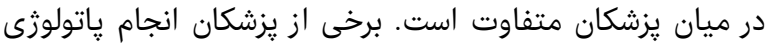

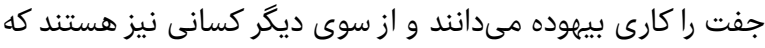

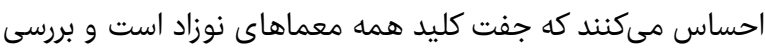

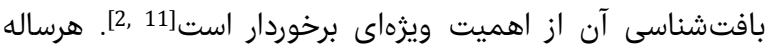

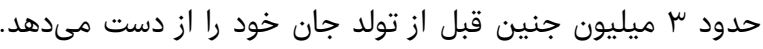

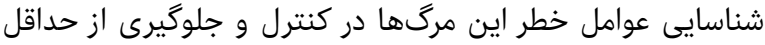

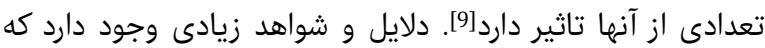

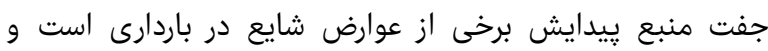

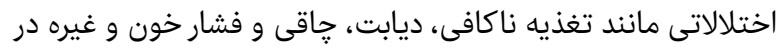

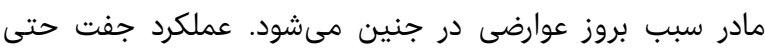

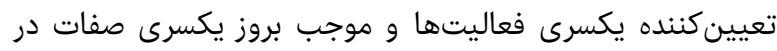

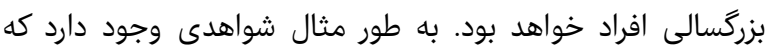

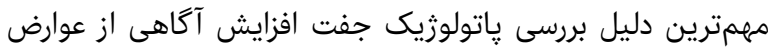

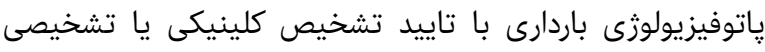

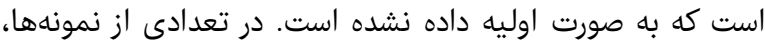

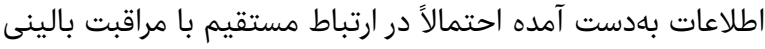

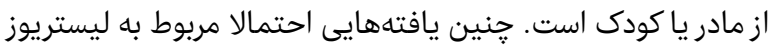

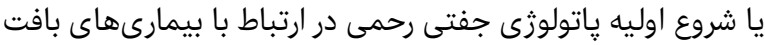

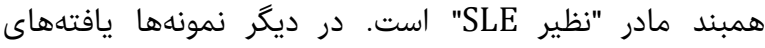

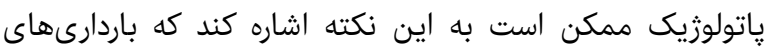

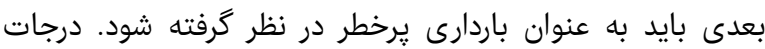

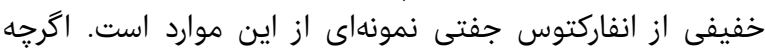

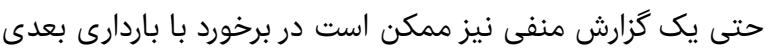

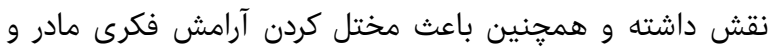
متخصص زنان شود.

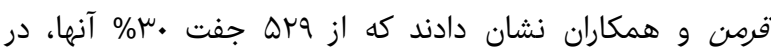

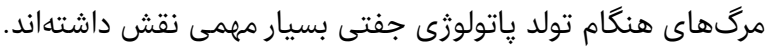

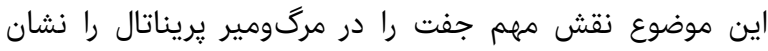

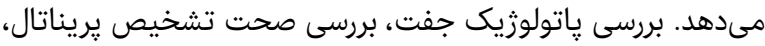

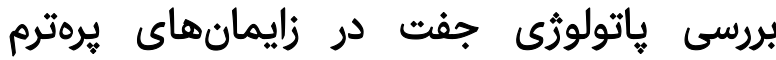

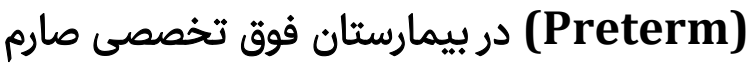

MD * عليرضا صرافزاده

مركز تحقيقات بارورى و نابارورى صارم، بيمارستان فوق تخصصى صارم، تهران، ايران

PhD محمد جمالو ائوان كروه فناورى اطلاعات، دانشكده مديريت، دانشكاه تهران، تهران، ايران

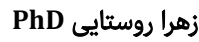

مركز تحقيقات بارورى و نابارورى صارم، بيمارستان فوق تخصصى صارم، تهران،

جكيده

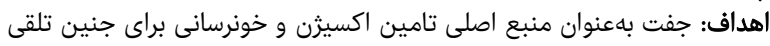

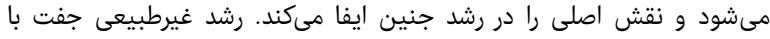

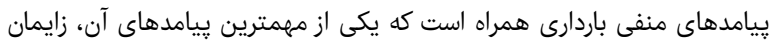

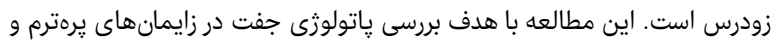

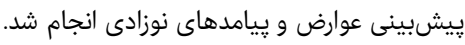

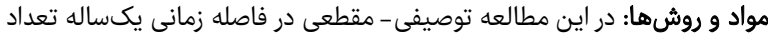

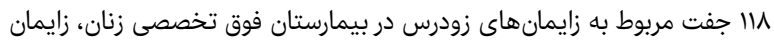

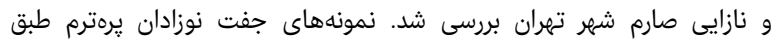

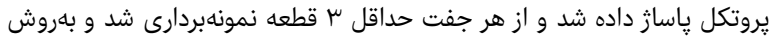

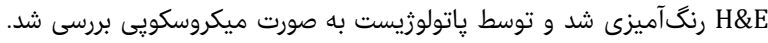

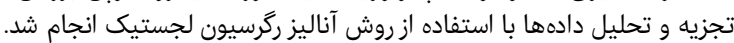

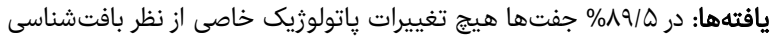

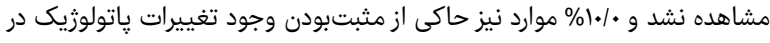

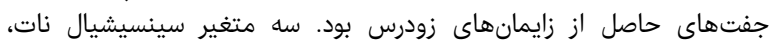

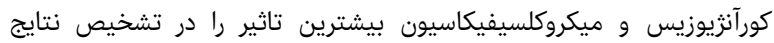

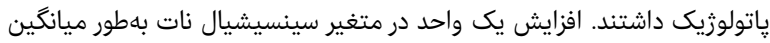

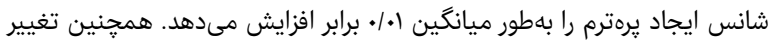

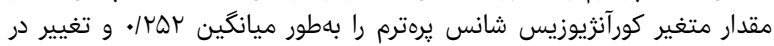

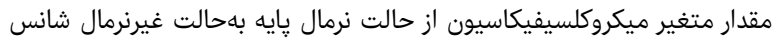

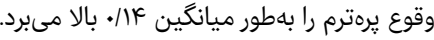

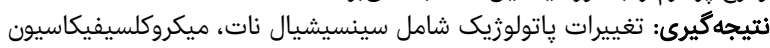

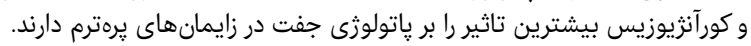

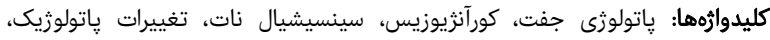
ميكروكلسيفيكاسيون، زايمان زودرس آنران

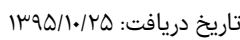

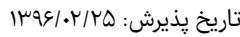
alirezasarrafzadeh@yahoo.com : نويسنده مسئون:

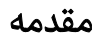

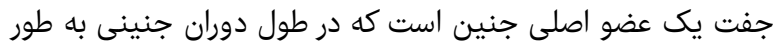

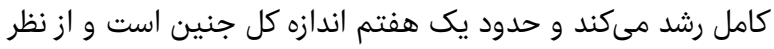

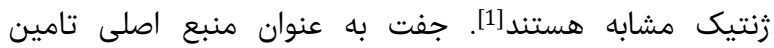

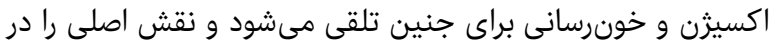

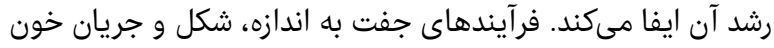

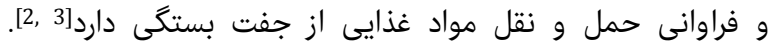

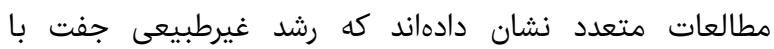

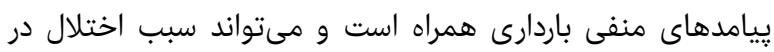

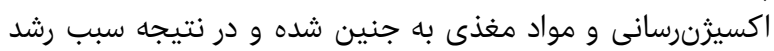

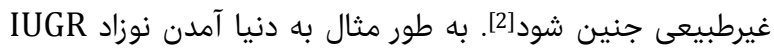

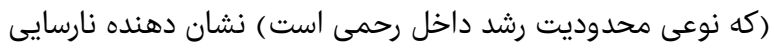

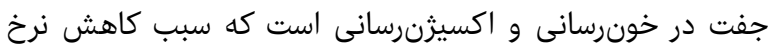

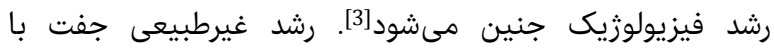

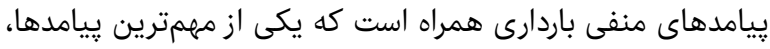

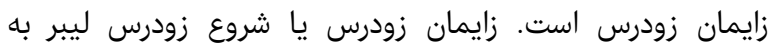




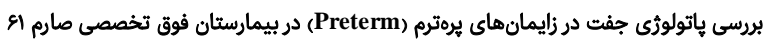

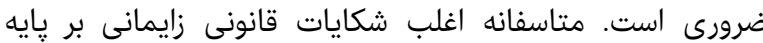

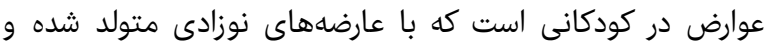

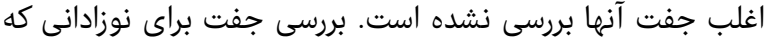

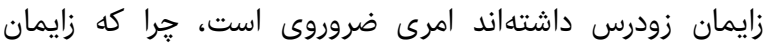

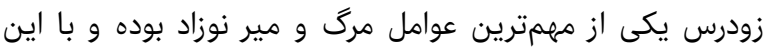

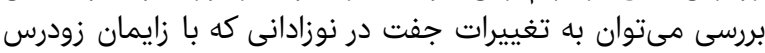

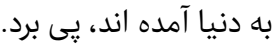

جدول () انديكاسيونهاى مادرى، جنينى/نوزادى و جفتى براى بررسى جفت

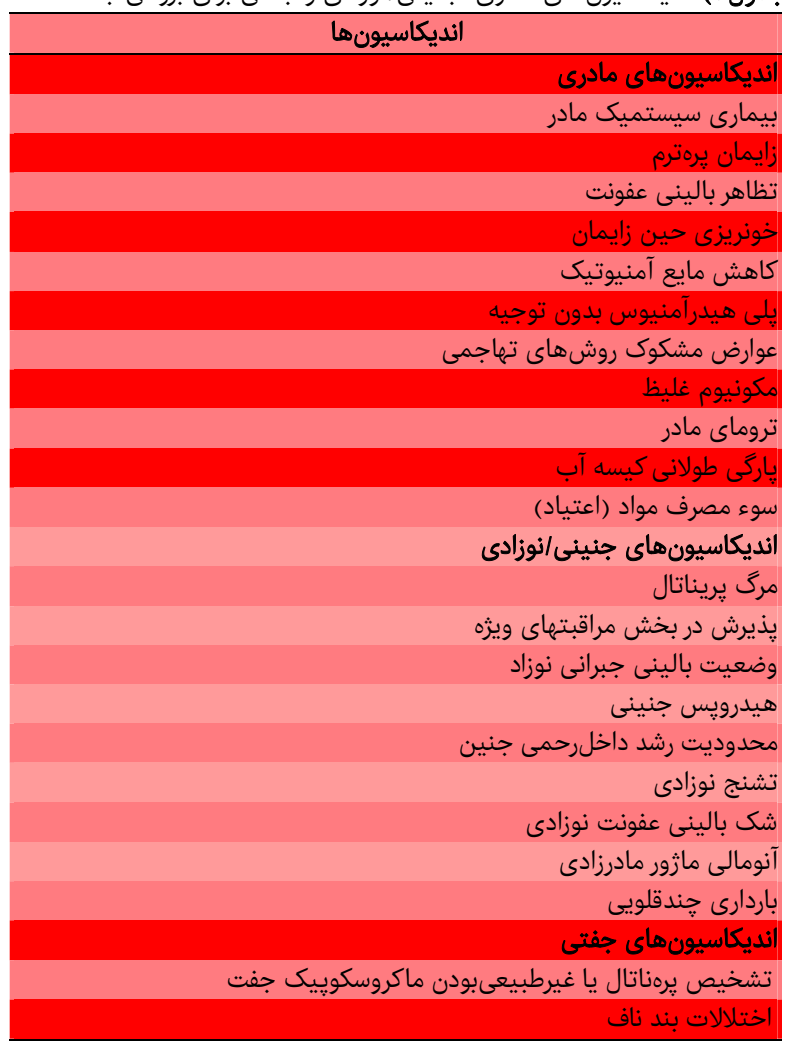

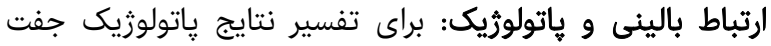

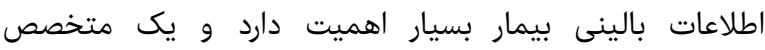

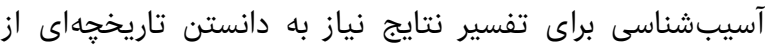

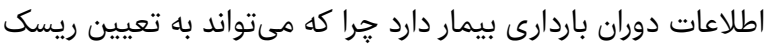

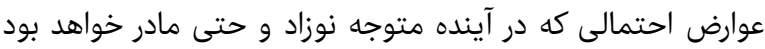

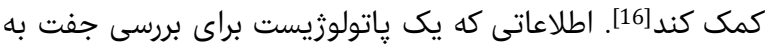

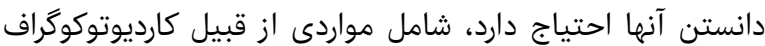

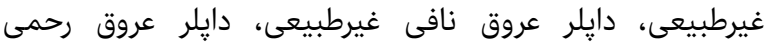

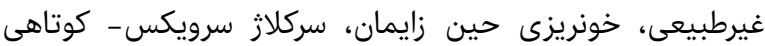

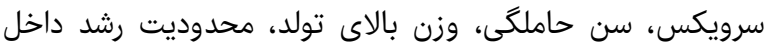

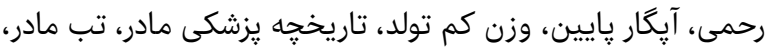

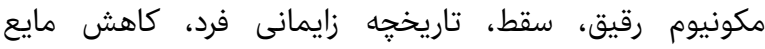

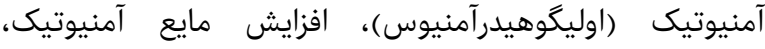

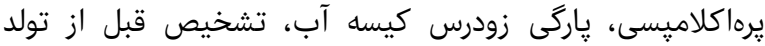

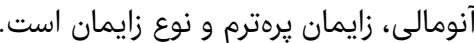

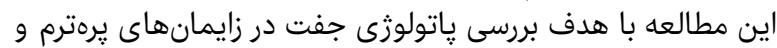

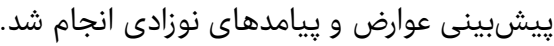

\section{مواد و روشها}

در اين مطالعه توصيفى- مقطعى در فاصله زمانى يك ساله
بررسى علل زايمان يرهترم و دستيابى جُندگًانه به مراقبتهاى

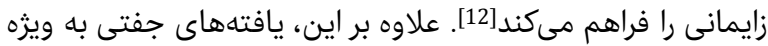

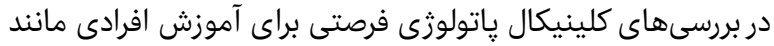

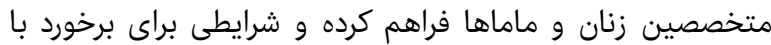

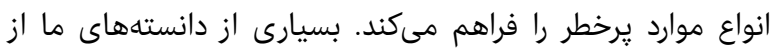

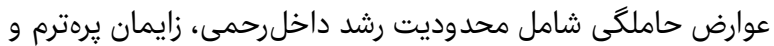

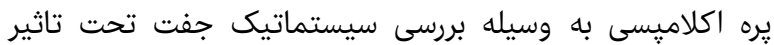

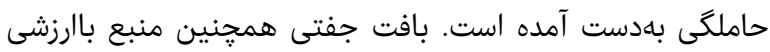

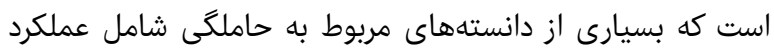

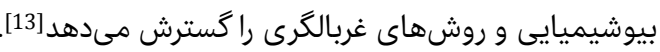

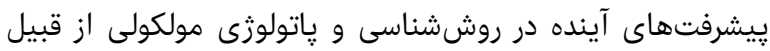

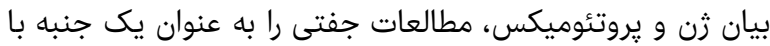

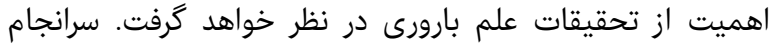

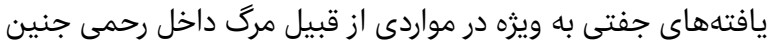

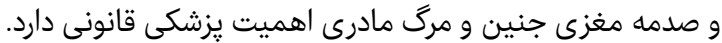

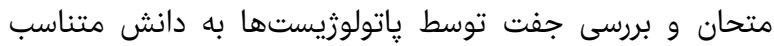

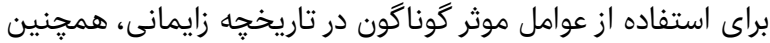

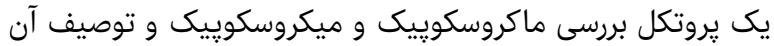

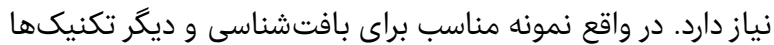

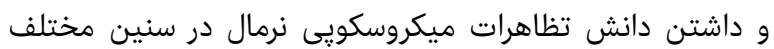

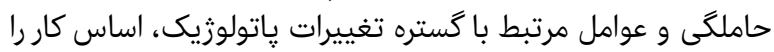

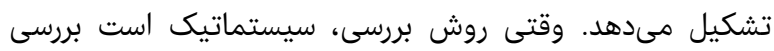

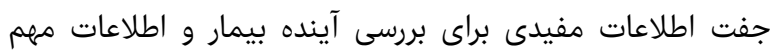

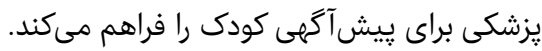

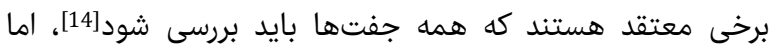

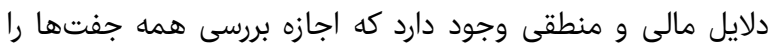

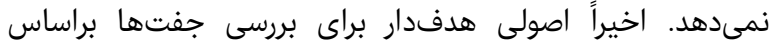

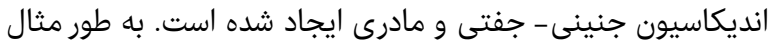

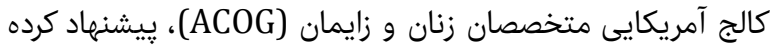

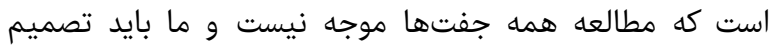

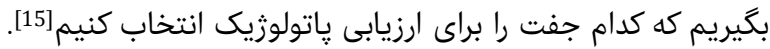

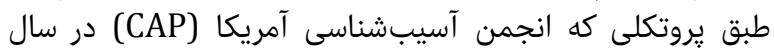

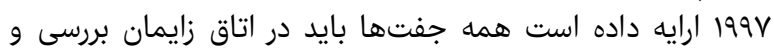

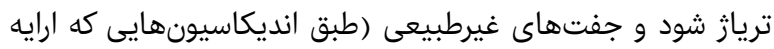

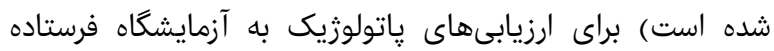

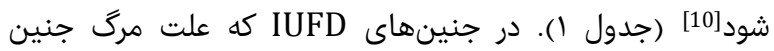

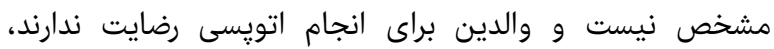

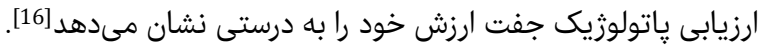

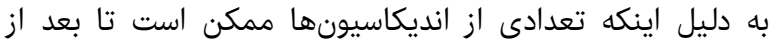

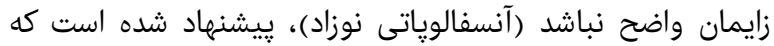

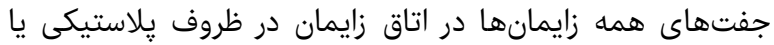

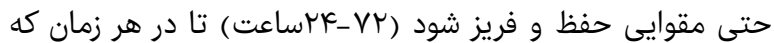

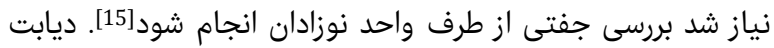

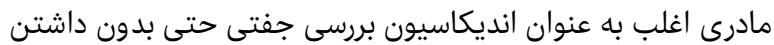

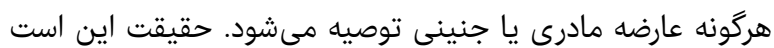

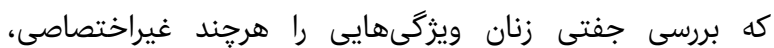
مشخص مىكند. در همه زنان حامله با بيمارى نئويلاستيك ضرورى است است كه جفت إنت

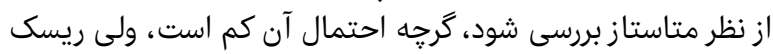

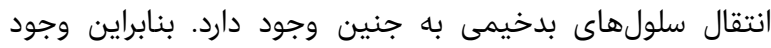

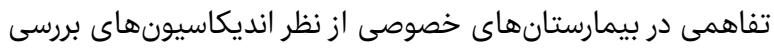
جفتى بين ياتولوزيست، متخصص زيمارسنان خصوصى ازنان و متخصص نديكاسون نوزادان 


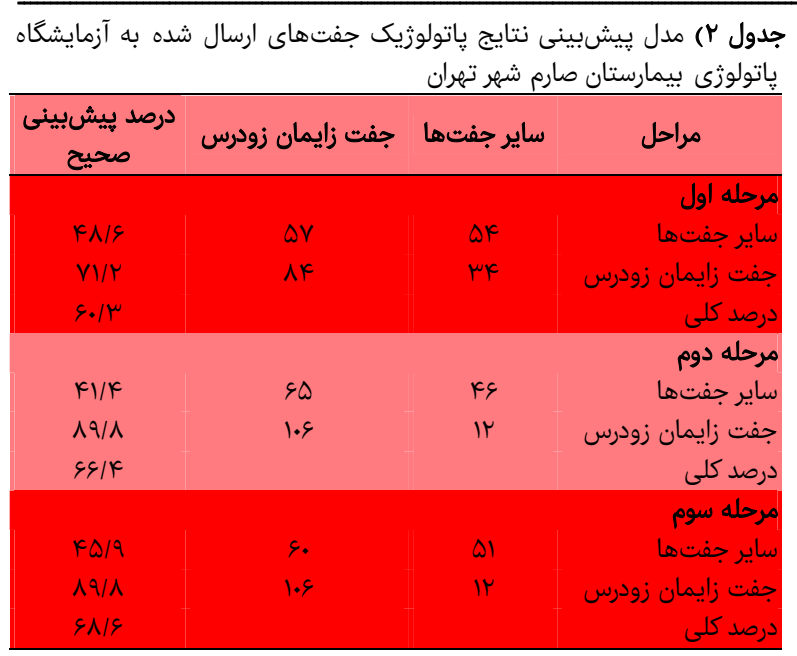

9. 1 جفت علتهاى ديكرى براى ارسال نداشتند و تنها علت ارسال

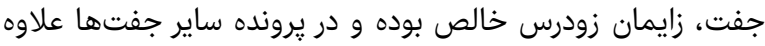

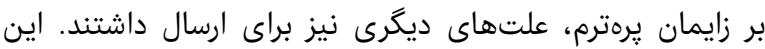

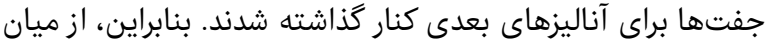

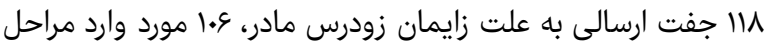

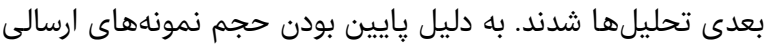

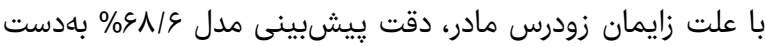

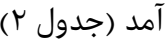

مدل رگرسيون لجستيك براى تحليل عردي عوامل موثر بر يرهترم شامل

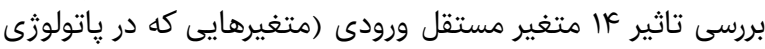

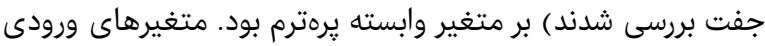

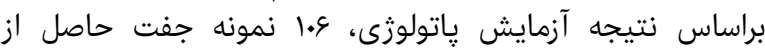
زايمانهاى زودرس بوده كه تنها دآنها دليل ارسال آنها به آزمايشكاه

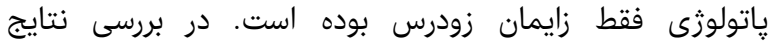

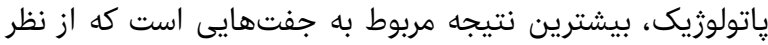

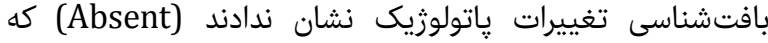

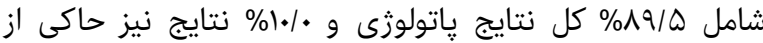

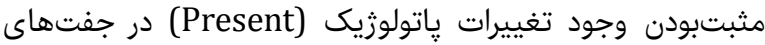

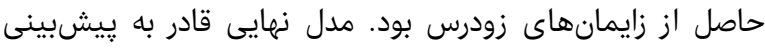

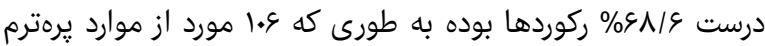

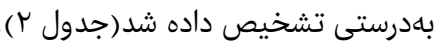

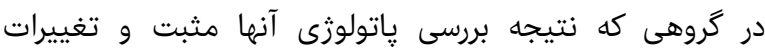

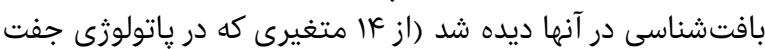

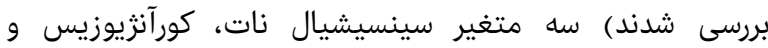

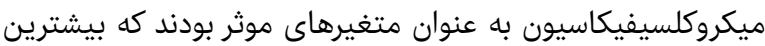

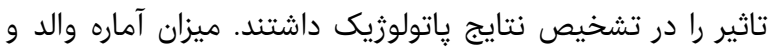

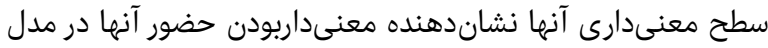

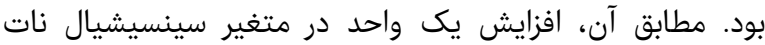
هانس ايجاد يرهترم را به طور ميانكين اء / • برابر بالا برد.

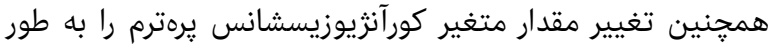

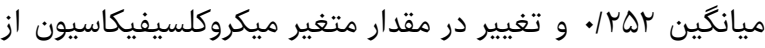

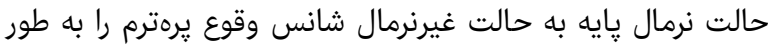

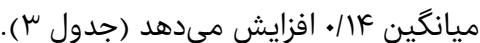

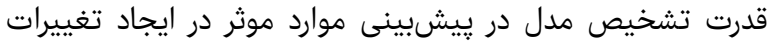

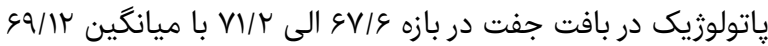

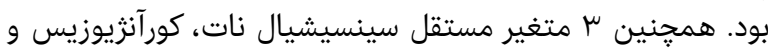

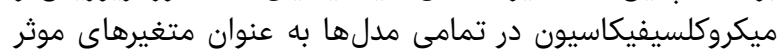

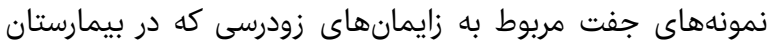

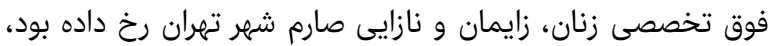

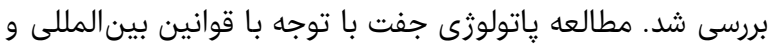

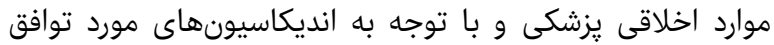

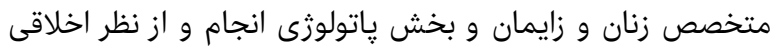

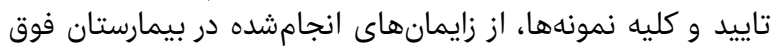

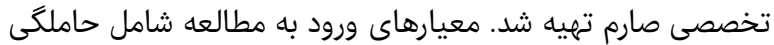

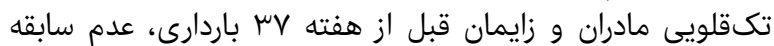

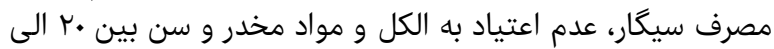

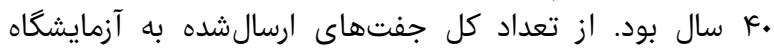

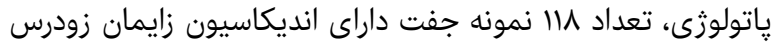

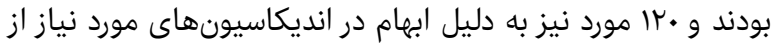

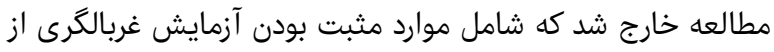

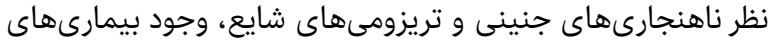

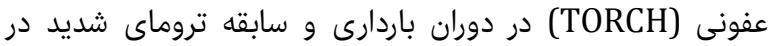

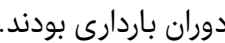

همه نوزادان در بدو تولد بررسى شدند و و با بآيكار مساوى V و بالاتر

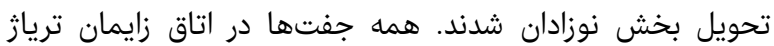

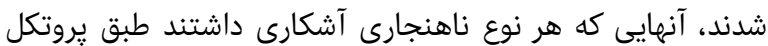

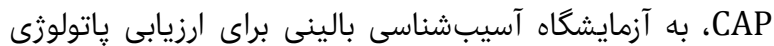

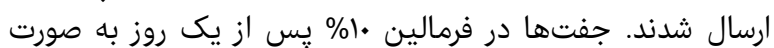

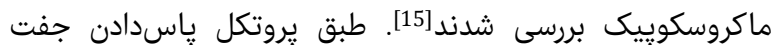

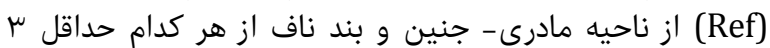

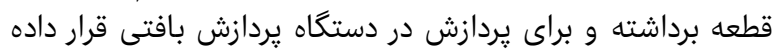

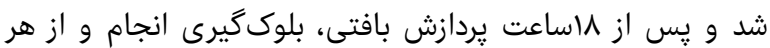

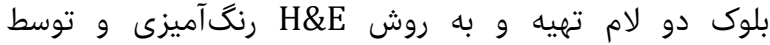

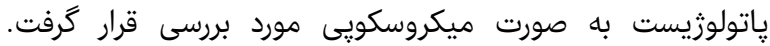

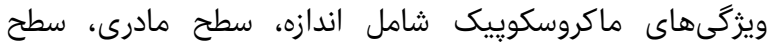

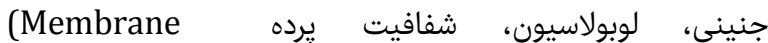
(Umbilical length) هماتوما و طول نافي فيكي (transparency)

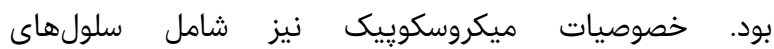

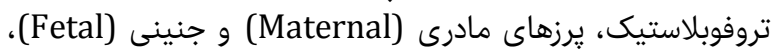
رسوب فيبرينوئيدى (Fibrinoid dep.)، التهاب موضعى (Focal) (Basal plate نكروز صفحه قاعدهاى (inflammation) (Fetal vessels in عروق جنينى در صفحه كوريونى، صفيرون، (necrosis) chorionic plate)

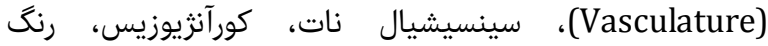
موكونيوم (Meconium staining) و ميكروكلسيفيكاسيون بودند. دادهها به روش تحليل رخرسيون لجستيك با روش انتخاب فيلد تجزيه و تحليل و به منظور انتخاب تعداد ركوردهاي انتياي

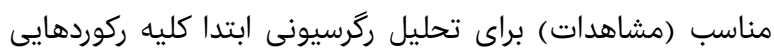

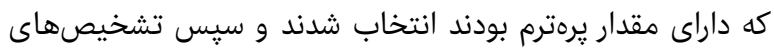

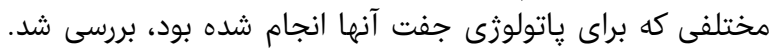

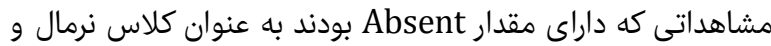

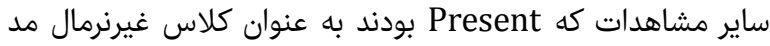

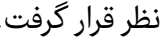

يافتهها

دادههاى تمام جفتهايى كه با علت يرةترم به آزمايشكاه يِاتولوزى

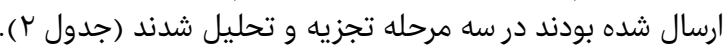




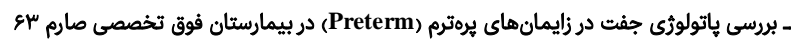
با توجه به معيارهاى ورود به مطالعه كه شامل عدم مصرف درف سيكار

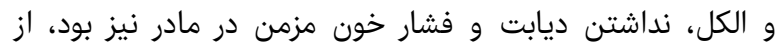

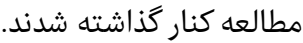

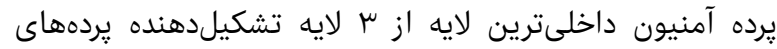

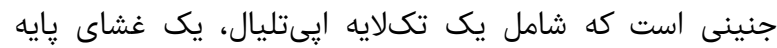

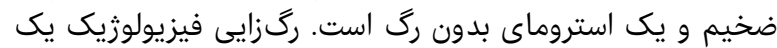

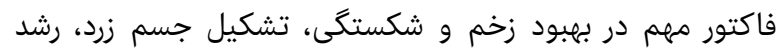

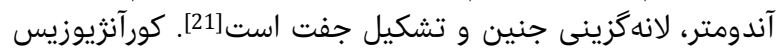

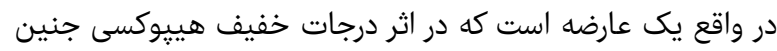

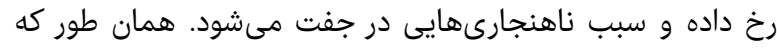

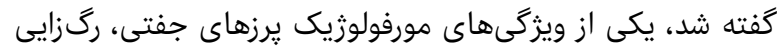

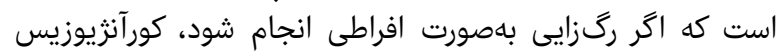
ناميده مىشود [22].

كره سينسيشيال به تجمع كانونى هستهاهـاى سينسيشيال در سطح

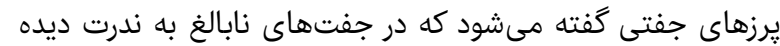

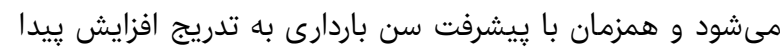

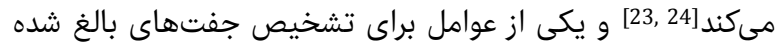

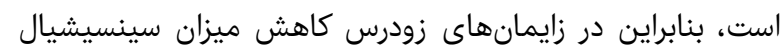
نات را شاهد هستيم.

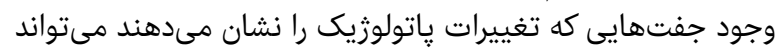

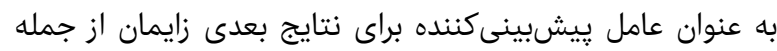

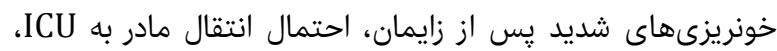

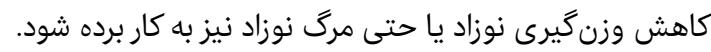

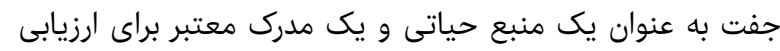

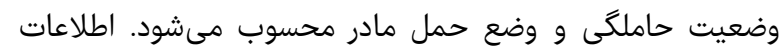

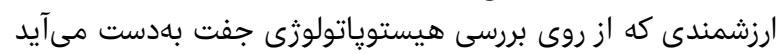

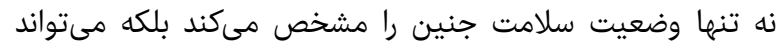

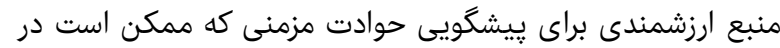

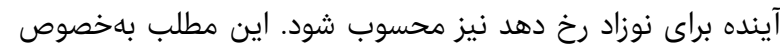

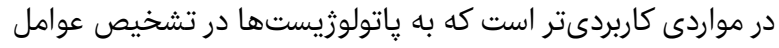

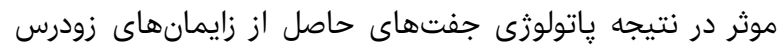
يارى مىرساند.

\section{نتيجه نيرى}

تغييرات پاتولوزيك شامل سينسيشيالنات، ميكروكلسيفيكاسيون

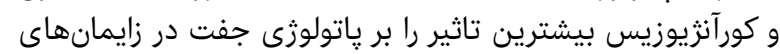

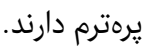

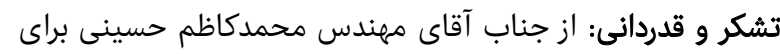

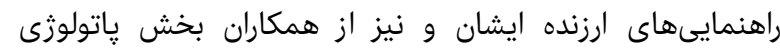
آزمايشگاه جنرال بيمارستان فوق إندان تخصصى صارم كمال تشكر و قدردانى را داريم.

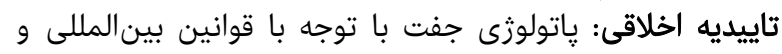

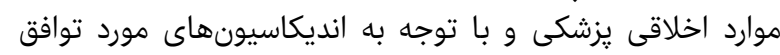

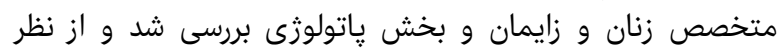

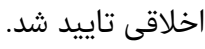

تعارض منافع: تعارض منافعى وجايد وجود ندارد.

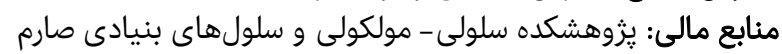

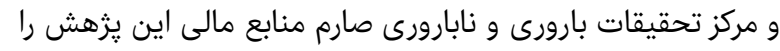
تامين نموده است.

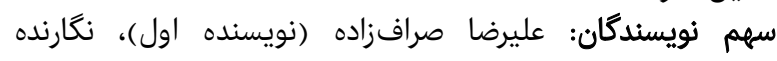

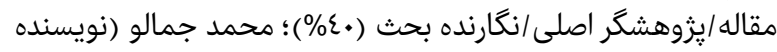

جدول س) بررسى متغيرهاى موثر در ايجاد تغييرات ياتولوزيك در جفت حاصل از

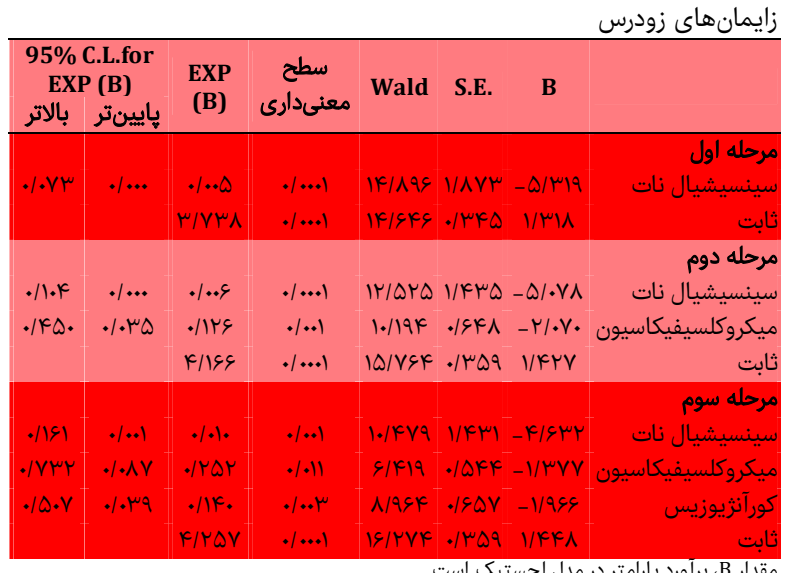

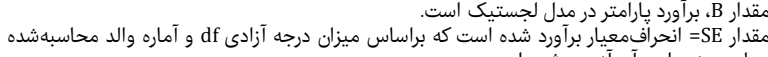
سطح معنى دارى آن آزمون شده است.

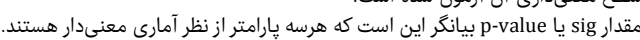

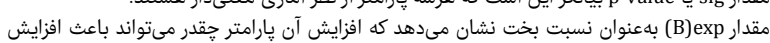
يا كاهش شانس وقوع فيلد هدف شود.

بحث

زايمان يرهترم يكى از انديكاسيونهاى مادرى بررسى جفت است.

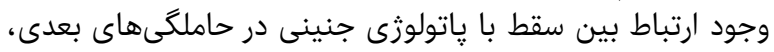

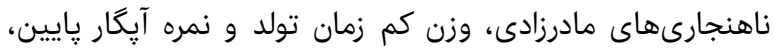

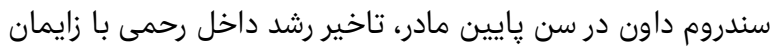

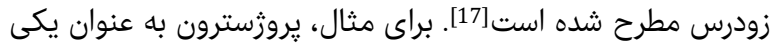

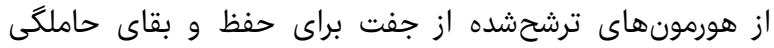

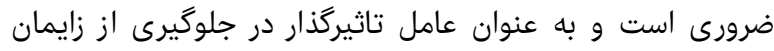

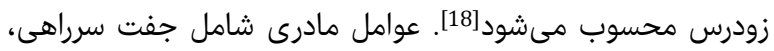

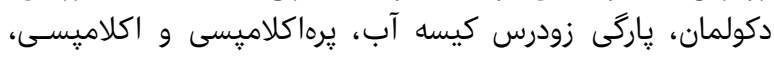

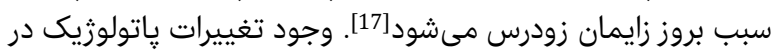

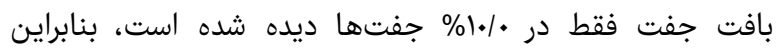

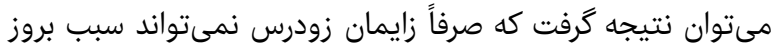

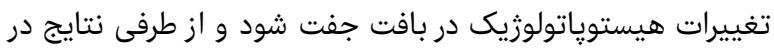

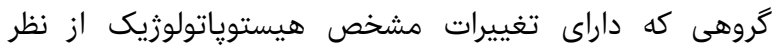

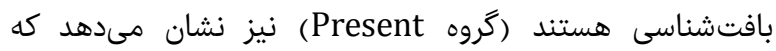

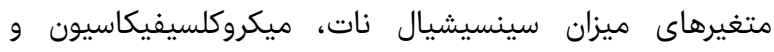

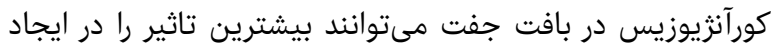

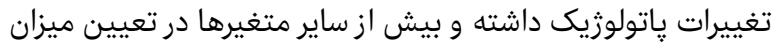

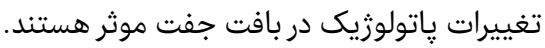

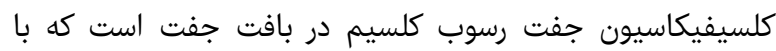

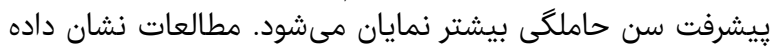

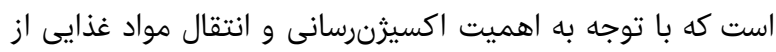

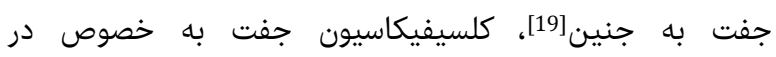

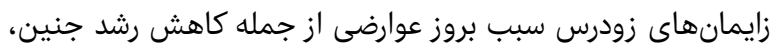

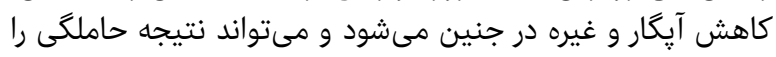

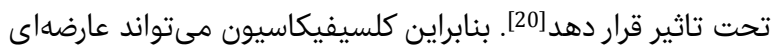

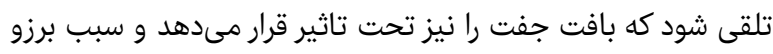

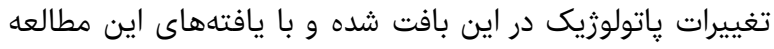

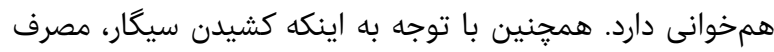

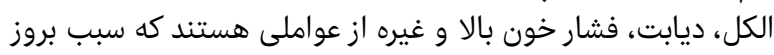

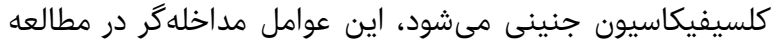


illness severity in preterm infants in the first twenty four hours after birth. Early Hum Dev. 2011;87(4):315-9.

12- Gherman RB, Incerpi MH, Wing DA, Goodwin TM. Ballantyne syndrome: Is placental ischemia the etiology? J Matern Fetal Med. 1998;7(5):227-9.

13- Finn JL. Placenta membranacea. Obstet Gynecol. 1954;3(4):438-40.

14- Salafia CM, Vintzileos AM. Why all placentas should be examined by a pathologist in 1990. Am J Obstet Gynecol. 1990;163(4 Pt 1):1282-93.

15- Sills A, Steigman C, Ounpraseuth ST, Odibo I, Sandlin AT, Magann EF. Pathologic examination of the placenta: Recommended versus observed practice in a university hospital. Int J Womens Health. 2013;5:309-12.

16- Gordijn SJ, Dahlstrom JE, Khong TY, Ellwood DA. Histopathological examination of the placenta: Key issues for pathologists and obstetricians. Pathology. 2008;40(2):176-9.

17- Paz JE, OTANO L, Gadow EC, Castilla EE. Previous miscarriage and stillbirth as risk factors for other unfavourable outcomes in the next pregnancy. $\mathrm{Br} \mathrm{J}$ Obstet Gynaecol. 1992;99(10):808-12.

18- Saghafi N, Khadem N, Mohajeri T, Shakeri MT, Amini M. Efficacy of $17 \alpha$-hydroxyprogesterone caproate in preterm delivery prevention of preterm delivary. J Obstet Gynaecol Res. 2011;37(10):1342-5.

19- Chen KH, Chen LR, Lee YH. The role of preterm placental calcification in high-risk pregnancy as a predictor of poor uteroplacental blood flow and adverse pregnancy outcome. Ultrasound Med Biol. 2012;38(6):1011-8.

20- Chen KH, Chen LR, Lee YH. Exploring the relationship between preterm placental calcification and adverse maternal and fetal outcome. Ultrasound Obstet Gynecol. 2011;37(3):328-34.

21- Niknejad H, Yazdanpanah G, Nikbin A, Tehrani F, Peirovi H. Effects of epithelial cells on amniotic membrane angiogenic properties using rat aortic ring assay. Koomesh. 2014;15(3):372-9.

22- Stanek J. Chorangiosis of Chorionic Villi: What Does It Really Mean?. Arch pathol Lab Med. 2016;140(6):588-93. 23- Jones C, Fox H. Syncytial knots and intervillous bridges in the human placenta: An ultrastructural study. J Anat. 1977;124(Pt 2):275-86.

24- Loukeris K, Sela R, Baergen RN. Syncytial knots as a reflection of placental maturity: Reference values for 20 to 40 weeks gestational age. Pediatr Dev Pathol. 2010;13(4):305-9.

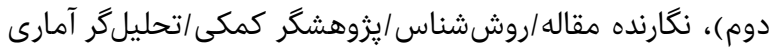

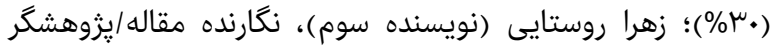

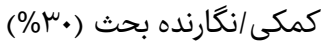

\section{منابع}

1- Lavery JP. The role of placental examination and its pathology in obstetric risk management. J Healthc Risk Manag. 1997;17(3):15-20.

2- Jafari H, Latifnejad Roudsari R. Horseshoe Placenta and Preterm Labour: A Case Report. J Midwifery Reprod Health. 2014;2(2):147-9.

3- Tufail S, Nawaz S, Sadaf M, Sial SS. Association between battledore placenta and perinatal complications. J Rawal Med Coll. 2012;16(2):159-61.

4- Lawn JE, Ketende KW, Cousens SN. Estimating the causes of 4 million neonatal deaths in the year 2000. Int J Epidemiol. 2006;35(3):706-18.

5- Ratzon R, Sheiner E, Shoham-Vardi I. The role of prenatal care in recurrent preterm birth. Eur J Obstet Gynecol Reprod Biol. 2011;154(1):40-4.

6- Beck S, Wojdyla D, Say L, Betran AP, Merialdi M, Requejo JH, et al. The worldwide incidence of preterm birth: A systematic review of maternal mortality and morbidity. Bull World Health Organ. 2010;88(1):31-8.

7- Sibai BM, Caritis SN, Hauth JC, MacPherson C, VanDorsten JP, Klebanoff M, et al. Preterm delivery in women with pregestational diabetes mellitus or chronic hypertension relative to women with uncomplicated pregnancies. The National institute of Child health and Human Development Maternal- Fetal Medicine Units Network. Am J Obstet Gynecol. 2000;183(6):1520-4.

8- Kramer MS, Wilkins R, Goulet L, Séguin L, Lydon J, Kahn SR, et al. Investigating socio-economic disparities in preterm birth: Evidence for selective study participation and selection bias. Paediatr Perinat Epidemiol. 2009;23(4):301-9.

9- Boskabadi H, Maamouri GA, Tabatabaie A, Ayati S, Hassanzadeh M, Davarnia M, et al. Study of the Incidence, and Maternal and fetal risk factors for intra uterine fetal death. J Mazandaran Univ Med Sci. 2015;24(122):33256.

10- Ventolini G, Ramesh S, Barhan S, Neiger R. Abnormal placental findings associated with non-reassuring fetal monitoring and excellent neonatal outcomes. Int J Clin Med. 2011;2(3):310.

11- Roescher AM, Hitzert MM, Timmer A, Verhagen EA, Erwich JJ, Bos AF. Placental pathology is associated with 